\title{
A discussion of the potential benefits to injury surveillance through inclusion of date of injury in hospitalisation data in New South Wales and
}

Australia

\author{
Andrew D. Hayen ${ }^{\mathrm{A}, \mathrm{C}}$, Soufiane Boufous ${ }^{\mathrm{A}}$ and \\ James E. Harrison ${ }^{\mathrm{B}}$ \\ ${ }^{\mathrm{A}} \mathrm{NSW}$ Injury Risk Management Research Centre, \\ University of New South Wales \\ ${ }^{\mathrm{B}}$ Research Centre for Injury Studies, Flinders University \\ ${ }^{\mathrm{C} C o r r e s p o n d i n g ~ a u t h o r . E m a i l: ~ a h a y e n @ h e a l t h . u s y d . e d u . a u ~}$
}

\begin{abstract}
Most hospitalised injury cases have sudden onset at a specific time and date, due to a well-defined external cause (eg, a road crash). Date of injury is not presently recorded in routine hospital separations data in NSW or Australia, though it is in New Zealand. Benefits of adding date of injury to the Inpatient Statistics Collection would include: more accurate estimation of the population incidence of serious injury; better assessment of the health system utilisation and costs attributable to injurious events; and better linkage of hospital data with other data relevant to injury measurement and control (eg, road crash data).
\end{abstract}

Injury has been identified as a health priority area in Australia, and is the subject of three national plans endorsed in July 2005 by Australian health ministers. ${ }^{1-3}$ Injuries leading to hospitalisation include the most serious non-fatal injuries, and incur substantial costs both to the health system and society. In 2001-02, injuries accounted for about $7 \%$ of all hospitalisations in Australia. ${ }^{4}$ Highquality information about the incidence of injury leading to hospitalisation, and trends in incidence, is needed, for example, to assess the burden of injury, determine priorities for injury prevention, guide and evaluate injury prevention and assist service planning.

However, in Australia, accurate estimates of the incidence of injuries leading to hospitalisation are not readily available from routinely collected data because of multiple counting of the same injuries in estimates of the incidence of injury.
In Australia, reports on injury indicators have indicated the potential value of including information on the date of injury in routinely collected hospitalisation data., ${ }^{5,6}$ In this paper we discuss the potential benefits of adding such a variable to hospitalisation data to improve surveillance of serious injury in Australia.

\section{Identification of incident cases of injuries leading to hospitalisation}

The Inpatient Statistics Collection (ISC) covers all inpatient separations from public and private hospitals in NSW. In NSW, all hospitals are required to submit details to the NSW Department of Health about every inpatient episode of care which ends with the discharge, transfer or death of the patient, or by the patient becoming a different type of patient, requiring transfer within the same hospital. All states in Australia maintain similar collections, which suffer from the problems we describe below.

When estimating incidence of injury in a population, each new case of injury should be counted only once. However, the number of injury-related 'separations' from hospital is not equivalent to the number of incident injury cases that result in hospitalisation, because some cases of injury result in more than one episode of in-hospital care, each of which ends with a 'separation' event. Hence, patients who have been readmitted or transferred from another hospital for treatment of the same injury are recorded more than once. This is particularly an issue for patients with serious injuries who are likely to have a series of readmissions for ongoing treatment or rehabilitation, for example, patients with extensive burns who require a number of skin grafts. $^{7,8}$ In New Zealand, which to our knowledge is the only jurisdiction that captures date of injury information in hospitalisation data, it has been estimated that about $9 \%$ of all inpatient episodes that were assigned an external cause code were readmissions, ${ }^{7}$ but accurate estimates are not available for Australia. Furthermore, the rate of readmission in New Zealand is not uniform across mechanisms of injury. ${ }^{7}$ In addition, patterns of coding of the ways in which episodes in hospital end (for example, as a discharge, transfer or type-change separation) may not be constant over time in Australian hospitalisation data, or between geographical areas. ${ }^{6}$ This further complicates 
assessment of trends and differences in population incidence of cases.

The ISC does not currently contain a unique patient identifier. However, probabilistic record linkage (in this case an 'internal' linkage of the ISC to itself) can be used to identify the same patient across different episodes of care. However, reliance on the use of a unique patient identifier (or an approximate identifier obtained through record linkage) to identify repeat admissions has some drawbacks. ${ }^{8}$ For example, a presumed repeat admission with the same diagnosis and external cause codes as the first admission may in fact be an admission for a new case of injury, such as a hip fracture on the other side of the injured person's body resulting from a fall, or a second episode of intentional self-poisoning. Probabilistic data linkage is limited as it relies heavily on ICD 10 -AM codes in the principal diagnosis field to distinguish between 'multiple admissions' for the same injury event and 'new admission(s)' of the same person for a different injury event.

In the absence of a unique personal identifier and date of injury, some studies ${ }^{9,10}$ have assumed that each discharge refers to a single episode of injury, thereby resulting in an overestimation of incidence rates. Others have used one or other of two variables, 'mode of separation' and 'mode of admission', which are available in Australian hospitalisation data, to identify and omit groups of records likely to be for second or subsequent inpatient episodes concerning the same injury. ${ }^{11}$ Excluding such records assumes that the subsequent separation will be recorded and also meet the study definition of injury (eg, requiring a principal diagnosis of injury). Also, these methods cannot account for readmissions following discharge home.

If used with a unique patient identifier or record linkage, a 'date of injury' field would simplify the identification of incident cases of injury-related hospitalisations. ${ }^{12}$ Even when a unique patient identifier is not available, use of probabilistic record linkage and date of injury will enable the period between the date of injury occurrence and admission to be determined. This would provide a useful indication of whether a particular hospital admission is likely to have been the first episode in hospital due to that injury incident.

\section{Identification of incident injury events occurring in hospitals}

A 'date of injury' item would also allow a distinction to be made between injuries that occur in the community and lead to initial admission and injuries that occur during the course of an episode in hospital (for example, a fall from a hospital bed). These cases are of special importance to health care services. Accurate data on these cases are vital to inform strategies designed to prevent injury in health care institutions, particularly hospitals.

Currently, the only indication of these cases is provided by the ICD-10-AM place of occurrence codes, ${ }^{13}$ which are not specific enough to provide an accurate picture of injuries that occur after admission to hospital. Place of occurrence codes are not able to distinguish between injury sustained in a hospital or in an outpatient clinic, nor between injury sustained in hospital by an admitted patient or a visitor. Although a 'Diagnosis onset type' variable (item 000773), designed to identify postadmission conditions, is included in the National Health Data Dictionary, ${ }^{14}$ it is not recorded in the ISC in NSW.

\section{Better record linkage of hospital data to other injury-related datasets}

A date of injury item would also be beneficial to any record linkage of hospital separations data to other sources that contain information on injury, such as road crash data collected by police and workers' compensation data. Such linkages of datasets are important for providing a comprehensive picture of the circumstances and causes of injury, and are useful for providing a better understanding of the outcomes of various injuries. For linkage of road crash data with hospital discharge data, for example, date of injury in the hospital discharge data would assist assessment of whether matched records are, in fact, true matches.

\section{Latency between injury and first admission}

In Australia, it is likely that admission to hospital for an injury takes places soon after the occurrence of the injury. However, this is not always the case, and those cases in which first admission is delayed may warrant special attention. This is because late admission might mark instances of injuries not usually requiring admission that have resulted in complications, or injuries occurring in circumstances (geographical or social) leading to late presentation to hospital. Inclusion of a 'date of injury' variable would allow direct calculation of the latency between injury and first admission.

\section{Conclusion}

Prevention needs to be guided by a clear understanding of the burden of, and causal factors associated with, injury. Information about the date of injury is critical for linking these to relevant exposures, most of which are acute in nature. The availability of a 'date of injury' item in the New Zealand hospital discharge data has allowed accurate identification of first admissions for injuries, injuries occurring in health care institutions and improved injury surveillance. ${ }^{7,12}$ The introduction of such a variable to NSW and Australian hospitalisation data would result in similar outcomes and enhance the value of the data used to support injury prevention and control in NSW and Australia. 


\section{References}

1. National Public Health Partnership (NPHP). The national injury prevention and safety promotion plan: 2004-2014. Canberra: NPHP, 2004.

2. National Public Health Partnership (NPHP). The national Aboriginal and Torres Strait Islander safety promotion strategy. Canberra: NPHP, 2004.

3. National Public Health Partnership (NPHP). The national falls prevention for older people plan: 2004 onwards. Canberra: NPHP, 2004.

4. Australian Institute of Health and Welfare. Australia's health 2004. Canberra: AIHW, 2004.

5. Commonwealth Department of Health and Family Services and Australian Institute of Health and Welfare. National health priority areas report: injury prevention and control 1997. AIHW Cat. No. PHE 3. Canberra: DHFS and AIHW, 1998.

6. Harrison JE, Steenkamp M. Technical review and documentation of current NHPA injury indicators and data sources. Injury Research and Statistics Series Number 14. Adelaide: AIHW, 2002.

7. Langley J, Stephenson S, Cryer C, Borman B. Traps for the unwary in estimating person based injury incidence using hospital discharge data. Inj Prev 2002; 8: 332-7. doi:10.1136/ip.8.4.332
8. Boufous S, Williamson A. Reporting of the incidence of hospitalised injuries: numerator issues. Inj Prev 2003; 9: 370-5. doi:10.1136/ip.9.4.370

9. Fingerhut LA, Warner M. Injury chartbook. Health, United States, 1996-97. Maryland: National Center for Health Statistics, 1997.

10. Schmertmann ML, Williamson A. NSW injury profile: a review of injury data (1995-1999) and identification of areas requiring further study. Sydney: NSW Injury Risk Management Research Center, 2002.

11. Population Health Division. The health of the people of New South Wales: report of the Chief Health Officer, 2004. Sydney: NSW Department of Health, 2004.

12. Alsop JC, Langley JD. Determining First Admissions in a Hospital Discharge File via Record Linkage. Meth Inform Med 1998; 37: 32-7.

13. National Centre for Classification in Health. The international statistical classification of diseases and related health problems, 10th revision, Australian modification (ICD-10-AM). Volume 5: Australian Coding Standards. 2nd ed. Sydney: NCCH, 2000.

14. Australian Institute of Health and Welfare. National Health Data Dictionary. Version 8.0. Canberra: Australian Institute of Health and Welfare, 1999. 